\title{
A brief review of functionally graded materials
}

\author{
Abdolreza Toudehdehghan ${ }^{1,2, *}, J . W . \operatorname{Lim}^{1}, K$. E. Foo ${ }^{1}$, M.I.N Ma'arof ${ }^{1}$, and J. Mathews ${ }^{1}$ \\ ${ }^{1}$ Department of Mechanical Engineering, INTI International University, Jalan BBN 12/1, Bandar \\ Baru Nilai, 71800 Nilai, Negeri Sembilan, Malaysia \\ ${ }^{2}$ Department of Mechanical Engineering, Universiti Tenaga Nasional , km7, Jalan Kajang-Puchong, \\ 43009 Kajang, Selangor, Malaysia.
}

\begin{abstract}
Material engineering has become one of the corner stones in various engineering applications. The scientific alteration of base materials into various inorganic and organic compounds has resulted in the development of Functionally Graded Material (FGM). FGM can be described as a cutting edge material in which the particular material's properties could be made varied as the dimension changes. The aims of this study was to review on FGM's applications and its manufacturing processes. In addition, the mathematical idealization of the FGM was also reviewed. Based on the review, since FGMs are highly heterogeneous, it can be idealized as their mechanical properties changes smoothly with respect to their spatial coordinates. Nonetheless, in order to analyze FGMS in an efficient manner, the homogenization schemes are needed to be simplified from their complicated heterogeneous microstructures. Closed-form solutions of some fundamental solid mechanics' problems can be obtained by this idealization. Conclusively, FGM is unique in relation to any of the individual materials that structures it. Suggestively, this provide a limitless possibility for future engineering applications.
\end{abstract}

\section{Introduction}

Recently, distinct materials are often integrated together and used as a part of structural components. This is commonly associated with complex or demanding engineering applications. Hence, in reducing the local stress concentrations initiated by sudden changes in material properties, the idea of functionally graded materials (FGMs) was proposed in Japan during the mid-1980s. Via the used of FGM, the results had shown significant decrease in the severity of interfacial stresses whilst showing the conservation of structural quality and ductility. This key advantage given by FGM is highly beneficial to various present day endeavours. This is especially true in developing new materials for applications related to biomedical gadgets /prosthetics, solar panels, aviation transport, sensors and ceramics. It should be noted however, for FGM, the production process requires a metric as a method for controlling performance specifications. Spatial deformities in the compound structure or physical properties of a material volume will affects the FGM's performance. Therefore, in overcoming this issue, modern coatings are produced to create a homogeneous surface with

\footnotetext{
*Corresponding author: abdolreza.toudehdehghan@newinti.edu.my
} 
the objective of limiting potential failure areas. This concept is the reasoning behind systematic efforts to define and characterize determinations or specifications for FGM. Conventionally, the FGM are manufactured in using a blend of metals and ceramics and are characterized in a way that composition of each and the volume division of materials are changed gradually. Therefore, with the gradual fluctuation of the volume fraction on the constituent materials, the particular material properties show a smooth and nonstop change starting with one surface then onto the next.

\section{Production methods of functionally graded coating}

Conventionally in the industry, the surface thin layer FGMs is manufactured via coating methods. In this section, several coating methods are reviewed.

\subsection{Power Metallurgy (PM)}

Powder metallurgy (PM) is commonly utilized in the industry in order to deliver practically graded material [1] via the following three (3) steps: (i) weighing and blending of powder as indicated by the pre-composed spatial distribution as dictated by the functional prerequisite, (ii) stacking and ramming of the premixed-powders, (iii) sintering. In short, the PM strategy gives ascend to a stage wiser structure. Even so, if a nonstop structure is required to be achieved or desired, the centrifugal technique is utilized instead.

\subsection{Vapor deposition technique}

There are multiple versions of the vapour deposition techniques. Examples of these techniques are Chemical Vapour Deposition (CVD), Physical Vapour deposition (PVD) and sputter deposition, among others. These vapour deposition techniques are utilized to ensure slim surface covering and high-quality microstructure finish. Yet, these techniques produce harmful gases by-products [2]. Hence, precaution and safety measures need to be undertaken if the Vapor Deposition Technique is chosen as the manufacturing methods.

\subsection{Centrifugal methods}

The centrifugal method is similar to centrifugal casting where the gravity force is used through turning of the mold to frame mass functionally graded material [3]. The graded material is created via the distinction with respect to the materials' densities and the spinning of the mold. It should be noted that consistent reviewing in grading can be accomplished via the centrifugal method. Nevertheless, this method is limited only to circular and hollow shapes. Another issue in relation to the centrifugal method is that there is limiting point to specification of gradient produced [4] since the gradient is formed through common process (centrifugal force and thickness difference). Therefore, in understanding and overcoming these problems, researchers are practicing alternative manufacturing techniques such as the Solid Free Form (SFF).

\subsection{Solid Free Form (SFM) fabrication method}

Solid free form is an added substance fabricating process that offers loads of points of interest. Examples of these characteristics of interest are the capacity to create complex shapes, lesser energy, high material usage, higher speed of generation, and design freedom as parts are delivered straightforwardly from CAD (e.g. AutoCAD) data [5]. There are various types of SFF innovations, even so, the laser based forms are mostly utilized in creating functional graded material [6]. Laser based SFF process for FGM [7] include: (i) 
laser cladding based strategy $[8,9]$, and (ii) Selective Laser Sintering (SLS) $[10,11]$. SFF includes five essential steps [12]. The five steps are: (i) the generating of CAD information from the product like AutoCAD, Solid edge etc., (ii) the transformation of the CAD information to Standard Triangulation Language (STL) file, (iii) the slicing of the STL into two dimensional cross-area profiles, (iv) developing of the part layer by layer, and (v) expulsion and finishing. In short, the Solid Free Form (SFF) fabrication method is a great method in producing the FGMs.

\subsection{Other methods in FGMs fabrication}

Aside from the aforementioned methods, there are several other methods which are commonly practiced in the industry in order to produce the FGMs. Examples of these techniques are the electro-deposition, plasma spraying, electrophoretic, Ion Beam Assisted Deposition (IBAD), Self-Propagating, High-temperature Synthesis (SHS) [3]. Nonetheless, these methods cannot be used to create mass FGM since these methods requires longer manufacturing duration and energy intensive. Henceforth, these methods are uneconomical to be utilized as a part of delivering mass FGM.

\section{Field of application of FGM}

These days, in view to the capacity to produce composite materials which in accordance to functional stability and application requirement, the scope of FGM utilization is exceptionally wide. The distinctive ranges of FGM utilization is summarized in Figure 1.

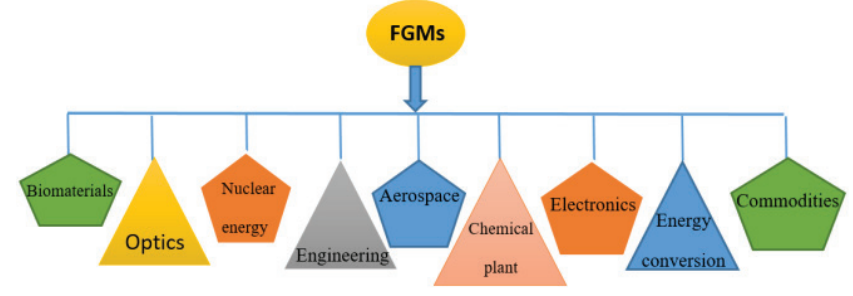

Fig.1 Schematic field of FGMs applications

For medical application, FGMs are used to supplant for naturally occurring FGM such as bones and teeth [13]. Hence, FGMs are extensively used in dental and orthopedic applications for teeth and bone replacement [14]. Whereas in nuclear projects, FGMs are widely used where great thermal opposing material is required. This is usually with respect to having a certain structure being sealed in order to maintain a strategic distance from modern mishaps which happens because of combination reaction process. For similar purposes, the FGM is utilized as defensive covering on turbine edges in gas turbine motor. Since FGMs can withstand high thermal gradient, this makes it appropriate for use in rocket engine components, structures and space plane body [15]. For optoelectronic applications, FGMs are used as graded refractive list material and in audio -videodiscs having magnetic capacity as storage media. Closely related to this field is the utilization of FGMs are in the communication field. The FGMs are used for optical fiber wires, which require adequate electrical resistor at external cover and adequate electric transistor material property on inward side. In regards to the defense industry, the FGMs are utilized in safeguarding application of penetration resistant material used for shield plates and bullet - proof vest [16] due to its capacity to hinder crack propagation. Other miscellaneous used of the FGMs are as lenses points and in the semiconductors industry. In short, the FGMs could be utilized in various fields and industries. With more extensive research on this particular material, the scope of usage could be further expanded. 


\section{Mathematical idealizations of FGMs}

Since FGMs are highly heterogeneous, it can be idealized as continua with their mechanical properties changing smoothly with respect to the spatial coordinates. To analyze FGMS in an efficient manner, the homogenization schemes are needed to simplify their complicated heterogeneous microstructures. Closed-form solutions of some fundamental solid mechanics' problems can be obtained by this idealization. It will help in evolving and developing related numerical models of the structures. A typical FGM often represents a particulate composite with a prescribed distribution of volume fractions of constituent phases [17]. The FGM properties are generally assumed by following gradation through the thickness in continuous manner.

\subsection{Exponential Law}

This particular idealization for FGM modeling is very common in the fracture mechanics studies. The uniform thickness $h$, the typical material properties ' $\mathrm{P}(\mathrm{z})$ ' at any point located at a distance ' $z$ ' from the reference surface is being modelled to this equation:

Where $\lambda=\frac{1}{2} \ln \left(\frac{P_{t}}{P_{b}}\right)$.

$$
P(z)=P_{t} \exp \left(-\lambda\left(1-\frac{2 z}{h}\right)\right)
$$

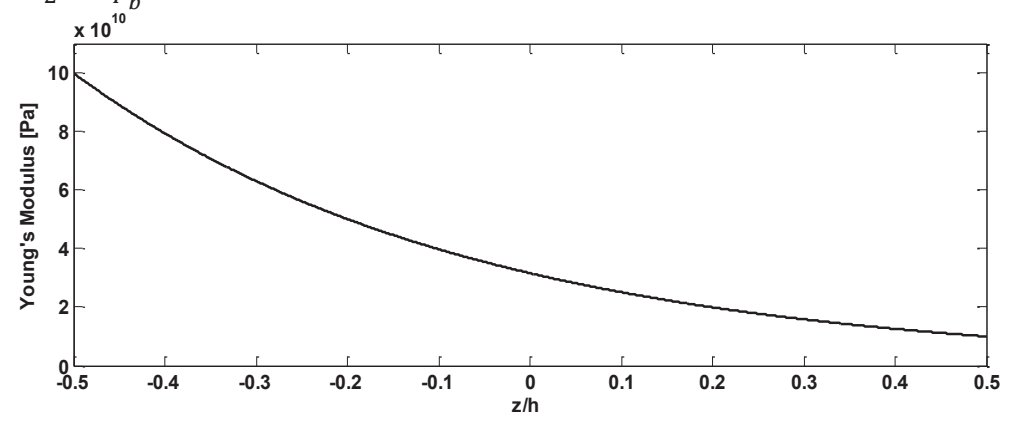

Fig.2 Exponential position dependence of elastic modulus of FGM layer

From figure 2, it is observable that the Young's modulus shows decrement as the $\mathrm{z} / \mathrm{h}$ value increases. Hence, adequate assessment is warranted to address this changes in material's mechanical properties. This is especially vital if the particular properties is related to design and/or safety issues.

\subsection{Exponential Law}

This is more common in the stress analysis of FGM $[17,18]$ and given by:

$$
P_{Z}=\left(P_{t}-P_{b}\right)\left(\frac{z}{h}+\frac{1}{2}\right)^{k}+P_{b}
$$

From the terminology, ' $\mathrm{P}(\mathrm{z})$ ' denotes a typical material property, viz., Young's modulus of elasticity (E), shear modulus of elasticity (G), Poisson's ratio (t), material density (q), etc. of the structures made of FGM [33]. ' $h$ ' is the total thickness of structure. ' $\mathrm{Pt}$ ' and ' $\mathrm{Pb}$ ' are the material properties at the top-most $(\mathrm{z}=+\mathrm{h} / 2)$ and bottom-most $(\mathrm{z}=-\mathrm{h} / 2)$ surfaces. ' $\mathrm{k}$ ' in the exponential model, and ' $\mathrm{k}$ ' in the power model are the material grading indexes respectively which depend upon the design requirements. 


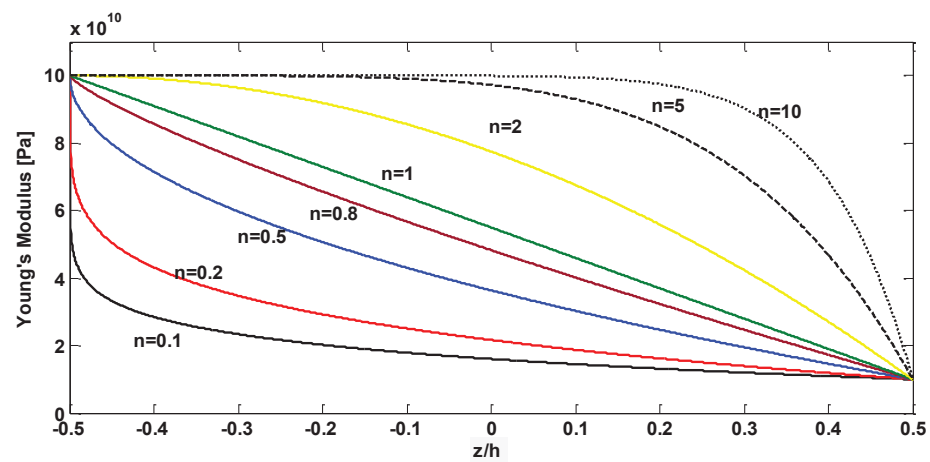

Fig. 3 Power-law position dependence of thermal expansion coefficient of FGM layer for several material parameters

From Fig. 3 it is observable as the $\mathrm{z} / \mathrm{h}$ value increases, the Young's Modulus characteristics changes with respect to the varying value of $n$. The $n=1$ line creates an inversing mirror image for the power-law position dependence of thermal expansion coefficient of the FGM layer.

\subsection{Sigmoid Function}

The volume fraction of the sigmoid function of FGM structures which is consisting of two power-law functions [19] can be written as below:

$$
\begin{gathered}
\mathrm{P}(\mathrm{z})=\left(P_{t}-P_{b}\right)\left(\frac{1}{2}\left(\frac{z}{h}-\frac{1}{2}\right)^{k}\right)+P_{b} \quad 0 \leq z \leq \frac{h}{2} \\
\mathrm{P}(\mathrm{z})=\left(P_{t}-P_{b}\right)\left(1-\frac{1}{2}\left(\frac{z}{h}+\frac{1}{2}\right)^{k}\right)+P_{b} \quad-\frac{h}{2} \leq z \leq 0
\end{gathered}
$$

Where $h, n$ are the thickness of the FGM layer and the material parameter respectively.

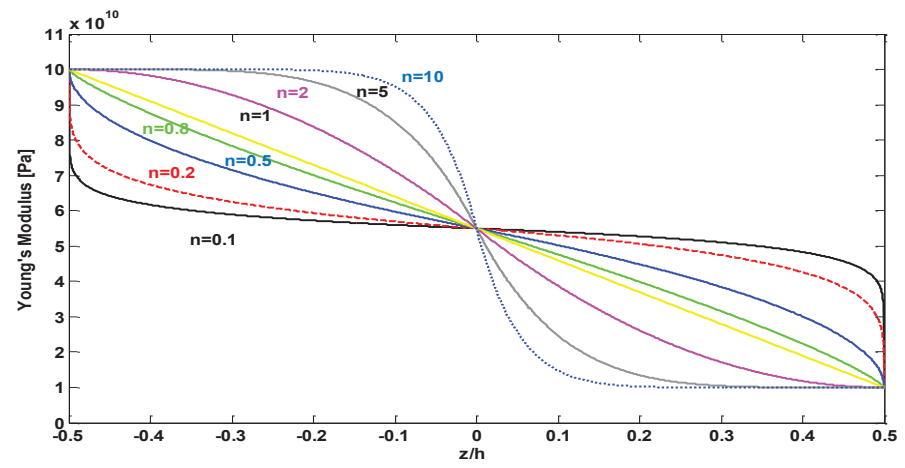

Fig.4 Sigmoid position dependence of Young's Modulus of FGM layer for several material parameters

As shown in Figure 4, at the top of layer is typically $\mathrm{Pb}$ and the bottom layer is typically Pt. Through the thickness of the FGM layer, there is a mixture of the two materials composition. From figure 4 it is observable that point 0 (center of thickness) is the converging and diverging point for the sigmoid position dependence of Young's Modulus of FGM layer for several material parameters.

\section{Conclusion}

Conclusively, FGM are one of a kind and unique in relation to any of the individual material that structures it. Suggestively, this provide a limitless possibility for future engineering applications. In order to analyze FGM in an efficient manner, the homogenization schemes 
are needed to simplify their complicated heterogeneous microstructures. Closed-form solutions of some fundamental solid mechanics' problems can be obtained by this idealization. It will help in evolving and developing related numerical models of the structures.

\section{References}

[1] M. M. Nemat-Alla, M. H. Ata, M. R. Bayoumi, and W. Khair-Eldeen, "Powder metallurgical fabrication and microstructural investigations of aluminum/steel functionally graded material," Materials Sciences and Applications, vol. 2, p. 1708, 2011.

[2] J. Groves and H. Wadley, "Functionally graded materials synthesis via low vacuum directed vapor deposition," Composites Part B: Engineering, vol. 28, pp. 57-69, 1997.

[3] Y. Watanabe, Y. Inaguma, H. Sato, and E. Miura-Fujiwara, "A novel fabrication method for functionally graded materials under centrifugal force: the centrifugal mixed-powder method," Materials, vol. 2, pp. 2510-2525, 2009.

[4] B. Kieback, A. Neubrand, and H. Riedel, "Processing techniques for functionally graded materials," Materials Science and Engineering: A, vol. 362, pp. 81-106, 2003.

[5] X. Lin and T. Yue, "Phase formation and microstructure evolution in laser rapid forming of graded SS316L/Rene88DT alloy," Materials Science and Engineering: A, vol. 402, pp. 294306, 2005.

[6] D. W. Hutmacher, M. Sittinger, and M. V. Risbud, "Scaffold-based tissue engineering: rationale for computer-aided design and solid free-form fabrication systems," TRENDS in Biotechnology, vol. 22, pp. 354-362, 2004.

[7] Y. T. Pei and J. T. M. De Hosson, "Functionally graded materials produced by laser cladding," Acta materialia, vol. 48, pp. 2617-2624, 2000.

[8] M. Ivosevic, R. Knight, S. Kalidindi, G. Palmese, and J. Sutter, "Solid particle erosion resistance of thermally sprayed functionally graded coatings for polymer matrix composites," Surface and Coatings Technology, vol. 200, pp. 5145-5151, 2006.

[9] K. A. Mumtaz and N. Hopkinson, "Laser melting functionally graded composition of Waspaloy ${ }^{\circledR}$ and Zirconia powders," Journal of materials science, vol. 42, pp. 7647-7656, 2007.

[10] M. Erdal, S. Dag, Y. Jande, and C. Tekin, "Manufacturing of functionally graded porous products by selective laser sintering," in Materials Science Forum, 2010, pp. 253-258.

[11] M. M. Gasik, "Functionally graded materials: bulk processing techniques," International Journal of Materials and Product Technology, vol. 39, pp. 20-29, 2010.

[12] M. A. Boboulos, CAD-CAM \& rapid prototyping application evaluation: Bookboon, 2010.

[13] W. Pompe, H. Worch, M. Epple, W. Friess, M. Gelinsky, P. Greil, et al., "Functionally graded materials for biomedical applications," Materials Science and Engineering: A, vol. 362, pp. 40-60, 2003.

[14] F. Watari, A. Yokoyama, M. Omori, T. Hirai, H. Kondo, M. Uo, et al., "Biocompatibility of materials and development to functionally graded implant for bio-medical application," Composites Science and Technology, vol. 64, pp. 893-908, 2004.

[15] L. Marin, "Numerical solution of the Cauchy problem for steady-state heat transfer in twodimensional functionally graded materials," International Journal of Solids and Structures, vol. 42, pp. 4338-4351, 2005.

[16] M. Malinina, T. Sammi, and M. M. Gasik, "Corrosion resistance of homogeneous and FGM coatings," in Materials Science Forum, 2005, pp. 305-310.

[17] H.-S. Shen, Functionally graded materials: nonlinear analysis of plates and shells: CRC press, 2016.

[18] R. Javaheri and M. Eslami, "Thermal buckling of functionally graded plates based on higher order theory," Journal of thermal stresses, vol. 25, pp. 603-625, 2002.

[19] Y.-L. Chung and W.-T. Chen, "Bending behavior of FGM-coated and FGM-undercoated plates with two simply supported opposite edges and two free edges," Composite Structures, vol. 81, pp. 157-167, 2007. 